\title{
On the ranks of configurations on the complete graph
}

\author{
Robert Cori $^{1}$ and Yvan Le Borgne ${ }^{12}$ \\ ${ }^{1}$ LaBRI, 351 cours de la Libération, F-33450 Talence, France \\ ${ }^{2}$ CNRS
}

\begin{abstract}
We consider the parameter rank introduced for graph configurations by M. Baker and S. Norine. We focus on complete graphs and obtain an efficient algorithm to determine the rank for these graphs. The analysis of this algorithm leads to the definition of a parameter on Dyck words, which we call prerank. We prove that the distribution of area and prerank on Dyck words of given length $2 n$ leads to a polynomial with variables $q, t$ which is symmetric in these variables. This polynomial is different from the $q, t$-Catalan polynomial studied by A. Garsia, J. Haglund and M. Haiman.

Résumé. Nous considérons le paramètre rang sur les configurations d'un graphes introduit par Baker et Norine . Nous nous intéressons plus particulièrement aux graphes complets et obtenons un algorithme efficace de déxtermination du rang d'une configuration pour ceux-ci. L'analyse de la complexité de cet algorithme conduit à définir un paramètre sur les mots de Dyck que nous appelons pré-rang. Nous démontrons que la distribution des aires et pré-rangs des mots de Dyck donne lieu à un polynôme à deux variables qui est symétrique en celles-ci. Il est différent du polynôme $q, t$-Catalan étudié par A. Garsia, J. Haglund et par M. Haiman.
\end{abstract}

Keywords: Rank, Riemann-Roch for graphs, Complete graphs, Dyck Words

We consider the following solitary game on an undirected connected graph with no loops: at the beginning a configuration $u$ is given, meaning that integer values $u_{i}$ are attributed to the $n$ vertices $x_{1}, x_{2}, \ldots x_{n}$ of the graph. These values can be positive or negative. At each step a toppling can be performed by the player on a vertex $x_{i}$ : it consists in subtracting $d_{i}$ (the number of neighbors of $x_{i}$ ) to the amount $u_{i}$ and adding 1 to all the amounts $u_{j}$ of the neighbors $x_{j}$ of $x_{i}$. In this operation the amount of vertex $x_{i}$ may become negative. The aim of the player is to find a sequence of toppling operations which will end with a configuration where all the $u_{i}$ are non negative. Since the sum $\operatorname{deg}(u)$ of the $u_{i}$ is invariant by the toppling, a necessary condition to succeed is that in the initial configuration $\operatorname{deg}(u)$ should be non negative.

This game has much to do with the chip firing game (see Björner et al. (1991), Biggs (1999)) and the sandpile model (see Bak et al. (1988), Dhar (1990), Dhar and Majumdar (1992)), for which recurrent configurations were defined and proved to be canonical representatives of the classes of configurations equivalent by a sequence of topplings.

The game was introduced and studied in detail by Baker and Norine (Baker and Norine (2007)) who introduced a new parameter on graph configurations: the rank. The rank $\rho(u)$ of a configuration $u$ is non negative if and only if one can get from $u$ a positive configuration by performing a sequence of topplings. 
For this parameter they obtain a simple formula expressing a symmetry similar to the Riemann-Roch formula for surfaces (a classical reference to this formula is the book by Farkas and Kra (1992)).

Our aim here is to study the values of this parameter when $G$ is the complete graph on $n$ vertices, for these graphs it was noticed (see Proposition 2.8. in Cori and Rossin (2000)) that the recurrent configurations correspond to the parking functions which play a central role in combinatorics. We obtain a simple greedy algorithm to compute the rank in that case, expected to be of linear complexity after optimisation, while there is no known polynomial time algorithm to compute that rank for arbitrary graphs.

The distribution of rank and degree on a natural subset of configurations over a graph $G$, the parking ones, is a bivariate power series $P_{G}(x, r)$ which has a symmetry inherited from the Riemann-Roch theorem. We show that some coefficients of these series are related to an evaluation of Tutte polynomial. In the case of complete graphs, we prove that our greedy algorithm to compute the rank has a linear complexity when assuming that arithmetic operations on the $u_{i}$ may be performed in constant time. Up to the classical action of symmetric group $S_{n}$ on configurations our algorithm may be described in terms of Dyck words. The analysis of this algorithm leads to the definition of a parameter on Dyck words, which will call prerank. We prove that the distribution of area and prerank on Dyck words of length $2 n$ leads to a polynomial in two variables which is symmetric in these. This polynomial has some values in common with the $q, t$-Catalan polynomial studied in Garsia and Haiman (1996); Haglund (2008). We provide a bijective proof of the symmetry of our polynomial and propose an expression for it using Tchebychev polynomials. Moreover the bistatistic prerank and dinv leads to the $q, t$-Catalan polynomial.

\section{Configurations on a graph}

\subsection{The Laplacian configurations}

Let $G=(X, E)$ be a multi-graph, where $X=\left\{x_{1}, x_{2}, \ldots, x_{n}\right\}$ is the vertex set and $E$ is a symmetric matrix such that $e_{i, j}$ is the number of edges with endpoints $x_{i}, x_{j}$, hence $e_{i, j}=e_{j, i}$. In all this paper $n$ denotes the number of vertices of the graph $G$ and $m$ the number of its edges. Moreover we suppose that $G$ is connected and has no loops, so that $e_{i, i}=0$ for all $i$.

We will consider configurations on this graph, which are elements of the discrete lattice $\mathbb{Z}^{n}$. Each configuration $u$ may be considered as assigning (positive or negative) tokens to the vertices. When there is no possibility of confusion the symbol $x_{i}$ will also denote the configuration in which the value 1 is assigned to vertex $x_{i}$ is and the value 0 is assigned to all others. Laplacian configurations $\Delta^{(i)}$ given by: $\Delta^{(i)}=d_{i} x_{i}-\sum_{i=1}^{n} e_{i, j} x_{j}$, where $d_{i}=\sum_{i=1}^{n} e_{i, j}$ is the degree of the vertex $x_{i}$, play a central role througout this paper.

The degree of the configuration $u$ is the sum of the $u_{i}$ 's and is denoted $\operatorname{deg}(u)$. We denote by $L_{G}$ the subgroup of $\mathbb{Z}^{n}$ generated by the $\Delta^{(i)}$, and two configurations $u$ and $v$ will be said toppling equivalent if $u-v \in L_{G}$, which will also be written as $u \sim_{L_{G}} v$.

\subsection{Parking configurations}

In each class of $\sim_{L_{G}}$ one configuration may be considered as a canonical representative. We call such configurations parking configurations since in the case of complete graphs, these are exactly the parking functions, a central object in combinatorics.

Definition 1 A configuration $u$ on a graph $G$ is a parking configuration if $u_{i} \geq 0$ for $i<n$ and for any subset $Y$ of $\left\{x_{1}, x_{2}, \ldots, x_{n-1}\right\}$ there is a vertex $x_{i}$ in $Y$ such that $u_{i}$ is less than the number of edges 
which have as endpoints $x_{i}$ and an $x_{j}$ not in $Y$. More precisely if there exists $i$ such that $x_{i} \in Y$ and $u_{i}<\sum_{x_{j} \notin Y} e_{i, j}$.

In other words a configuration $u$ is a parking configuration if and only if there is no toppling of all the vertices in a subset $Y$ of $\left\{x_{1}, x_{2}, \ldots x_{n-1}\right\}$ leaving all the $u_{i} \geq 0$.

Proposition 1 For any configuration u there exists a unique parking configuration denoted parking $(u)$ such that $u$ - parking $(u) \in L_{G}$

The proof of this Proposition is based on the notion of recurrent configurations which was considered and characterized by D. Dhar, a simple proof of the the uniqueness of a recurrent configuration is given in Cori and Rossin (2000).

\subsection{Parking configurations and acyclic orientations}

An orientation of $G$ is a directed graph obtained from $G$ by orienting each edge, so that one end vertex is called the head and the other vertex is called the tail. A directed path in such a graph consists of a sequence of edges such that the head of an edge is equal to the tail of the subsequent one.

The orientation is acyclic if there is no directed circuit, i.e. a directed path starting and ending at the same vertex. We associate to any parking configuration $u$ an acyclic orientation by:

Proposition 2 For any parking configuration u on $G=(X, E)$ there exists an acyclic orientation $\vec{G}$ such that for any vertex $x_{i}, i \neq n, u_{i}$ is strictly less than its indegree $d_{i}^{-}$.

Proof: We orient the edges using an algorithm that terminates after $n$ steps. Consider $Y=\left\{x_{1}, x_{2}, \ldots, x_{n-1}\right\}$. From the condition for parking configurations given above, there is one vertex $x_{i}$ such that $u_{i}<e_{i, n}$ then orient all these $e_{i, n}$ edges from $x_{n}$ to $x_{i}$, and remove $x_{i}$ from $Y$. Repeat the following operation until $Y$ is empty:

- Find $x_{k}$ in $Y$ such that $u_{k}<\sum_{x_{j} \notin Y} e_{k, j}$; orient all the edges joining any vertex $j$ outside $Y$ to $x_{k}$ from $x_{j}$ to $x_{k}$ and remove $x_{k}$ from $Y$.

\section{Effective configurations and rank}

In this section we give the main results of Baker and Norine (2007).

Definition 2 A configuration $u$ is positive if $u_{i} \geq 0$ for all $i$. A configuration $u$ is effective if there exists a positive configuration $v$ such that $u-v \in L_{G}$.

Since two equivalent configurations by $\sim_{L_{G}}$ have the same degree, it is clear that a configuration with negative degree is not effective. However we will prove that configurations with positive degree are not necessarily effective as the two examples in Figure 1(a) show.

\subsection{Configuration associated to an acyclic orientation of $G$}

Let $\vec{G}$ be an acyclic orientation of $G$, we define the configuration $u \vec{G}$ by: $(u \vec{G})_{i}=d_{i}^{-}-1$, where $d_{i}^{-}$is the number of edges which have head $x_{i}$. The configuration represented in Figure $1(\mathrm{~b})$ is equal to $u \vec{G}$ for the represented orientation of $G$.

Proposition 3 The configuration associated to an acyclic orientation of $G$ is non effective. 


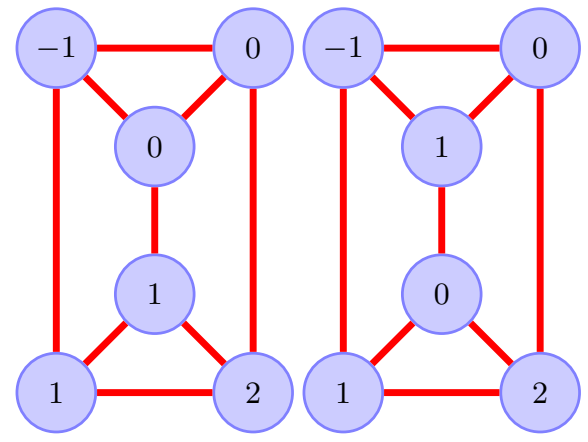

(a) An effective configuration (left) and a non effective one (right)

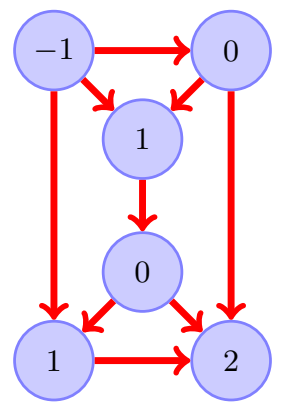

(b) An orientation of $G$ and the corresponding configuration

Fig. 1: Examples of effective, non-effective configurations and orientations.

\subsection{Characterisation of effective configurations}

Theorem 1 For any configuration u, one and only one of the following assertions is satisfied:

(1) $u$ is effective.

(2) There exists an acyclic orientation $\vec{G}$ such that $u \vec{G}-u$ is effective.

Moreover $u$ is effective if and only if the parking configuration $v$ such that $u \sim_{L_{G}} v$ satisfies $v_{n} \geq 0$.

Corollary 1 Any configuration u with degree greater than $m-n$ is effective.

Proof: If $u$ such that $\operatorname{deg}(u)>m-n$ is not effective, by the above theorem there exists an acyclic orientation $\vec{G}$ of $G$ such that $u \vec{G}-u$ is. But the degree of this configuration is negative, giving a contradiction.

\subsection{The rank of configurations}

From now on it will be convenient to denote positive configurations by using the letters $f, g \cdots$ and configurations with no particular assumptions on them by the letters $u, v, w$.

Definition 3 The rank $\rho(u)$ of a configuration is the integer defined by:

- If $u$ is non effective it is equal to -1

- If $u$ is effective, it is the largest integer $r$ such that for any positive configuration $f$ of degree $r$ the configuration $u-f$ is effective.

Denoting $\mathbb{P}$ as the set of positive configurations and $\mathbb{E}$ as the set of effective configurations, this definition can given by the following formula which is valid in both cases:

$$
\rho(u)+1=\min _{f \in \mathbb{P}, u-f \notin \mathbb{E}} \operatorname{deg}(f) .
$$

An immediate consequence of this definition is that if $\operatorname{deg}(u) \geq-1$ then $\rho(u) \leq \operatorname{deg}(u)$, and for any acyclic orientation $\vec{G}$ the rank of $u \vec{G}$ is -1 . Moreover if two configurations $u$ and $v$ are such that $u_{i} \leq v_{i}$ for all $i$ then $\rho(u) \leq \rho(v)$. 
Definition 4 A positive configuration $f$ is a prooffor the rank $\rho(u)$ of an effective configuration $u$ if $u-f$ is non effective and $u-h$ is effective for any positive configuration $h$ such that $\operatorname{deg}(h)<\operatorname{deg}(f)$.

Notice that if $f$ is a proof for $\rho(u)$ then $\rho(u)=\operatorname{deg}(f)-1=\operatorname{deg}(f)+\rho(u-f)$.

Proposition 4 A configuration $u$ of degree greater than $2 m-2 n$ has rank $r$ such that

$$
r+1=\operatorname{deg}(u)-(m-n) .
$$

Proof: We first show that for any positive configuration $f$ such that $\operatorname{deg}(f)=r$, the configuration $u-f$ is effective. This follows from $\operatorname{deg}(u-f)=\operatorname{deg}(u)-r=m-n+1$ by Corollary 1

We now build a positive configuration $f$ of degree $r+1$ such that $u-f$ is not effective. Consider any acyclic orientation $\vec{G}$ of $G$ and let $v=u-u \vec{G}$. Then $v$ is effective since its degree is equal to $\operatorname{deg}(u)-m+n$ and is therefore greater than $m-n$. Let $f$ be the positive configuration such that $v \sim_{L_{G}} f$, then $u-f$ is such that $u \vec{G} \sim_{L_{G}} u-v \sim_{L_{G}} u-f$ so that $u-f$ is not effective by Theorem 1

This result can be generalized into the following theorem which was given in Baker and Norine (2007) and called the Riemann-Roch theorem for graphs. A geometric interpretation of it is given in Amini and Manjunath (2010) and used in Manjunath (2011).

Theorem 2 Let $\kappa$ be the configuration such that $\kappa_{i}=d_{i}-2$ where for $i=1, \ldots, n$, the value $d_{i}$ is the degree of the vertex $x_{i}$. Then we have for any configuration $u$ :

$$
\rho(u)-\rho(\kappa-u)=\operatorname{deg}(u)-(m-n) .
$$

\section{A greedy algorithm computing the rank for configurations on complete graphs}

Configurations on the complete graph may be sorted in such a way that the first $n-1$ components form a weakly decreasing sequence. Clearly any configuration and its sorted version have equal ranks. The algorithm for determining the rank of $u$ that we will describe proceeds in a certain number of steps. Each of these steps consists in replacing $u$ by a $u^{\prime}$, and it will be convenient to work on their sorted versions. From an algebraic point of view this consists in considering orbits of the action of the symmetric group $S_{n-1}$ on the first $n-1$ components instead of mere configurations; the correctness of the computation is validated by the fact that all configurations in the same orbit have the same rank.

\subsection{Greedy algorithm on parking functions}

Any configuration $u$ is toppling equivalent to a single parking configuration parking $(u)$. In the case of the complete graph $K_{n}$ there is a linear time algorithm to compute it. It will be given below after developing the link between Dyck words and parking configurations. We first examine how to determine the rank of a parking configuration. On $K_{n}$, a configuration $u$ is a parking one if and only if after sorting the first $n-1$ entries one obtains $v=\left(v_{1}, \ldots v_{n-1}, u_{n}\right)$, satisfying $0 \leq v_{i}<n-i$ for any $1 \leq i<n$. In particular, $v_{n-1}=0$; so in any parking configuration at least one of the $u_{i}$ 's is equal to 0 . Our greedy algorithm determines the rank of a configuration $u$ on $K_{n}$ by iteratively computing the parking 
configuration $v$ equivalent to $u$ and subtracting 1 on one of the $v_{i}$ such that ${ }^{(\mathrm{i})} v_{i}=0$ until the resulting parking configuration is such that $u_{n}<0$. The rank is then equal to the number of iterations done, the algorithm is given in the left part of Figure 2 The fact that this algorithm correctly computes the rank is a consequence of the lemma below.

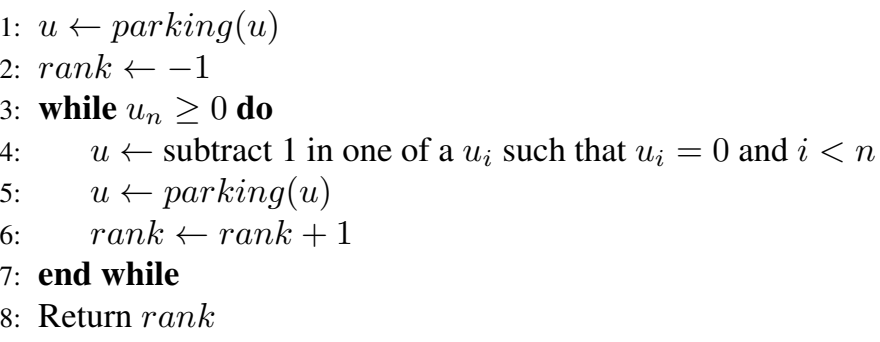

1: $u \leftarrow \operatorname{parking}(u)$

2: $(d, s) \leftarrow(d(u), s(u))$

3: $\operatorname{rank} \leftarrow-1$

4: while $s \geq 0$ do match $d$ with $a f b g$ $d \leftarrow g a b f$ rank $\leftarrow$ rank +1 $s \leftarrow s-|a f b|_{a}$

end while

10: Return rank

Fig. 2: Two versions of a greedy algorithm computing rank on $K_{n}$ : on configurations and Dyck words.

Lemma 1 Any positive configuration $u$ where $u_{i}=0$ admits a proof $g$ for its rank such that $g_{i}>0$.

Proof: Denote by $\epsilon^{(i)}$ the configuration where $\epsilon_{i}^{(i)}=1$ and for $j \neq i, \epsilon_{j}^{(i)}=0$. Let $f \geq 0$ be a proof of $\rho(u)$ and assume $f_{i}=0$, otherwise $g=f$ satisfies the lemma. Let $j \neq i$ such that $u_{j}-f_{j}=-a<0$. Let $v=u-\left(f-a \epsilon^{(j)}\right)$. Then $0 \leq f-a \epsilon^{(j)} \leq f$ and $v_{i}=0=v_{j}$. Let $\tau$ be the transposition which exchanges $i$ and $j$. Since $v=\tau v$, we have $g=f-a \epsilon^{(j)}+a \epsilon^{(i)}$ satisfies $g_{i}>0$, hence it is positive and has the same degree as $f$. Moreover $u-g$ is also non-effective since $u-g=v-a \epsilon^{(i)}=\tau$. $\left[v-a \epsilon^{(j)}\right]=\tau(u-f)$, hence $g$ is the proof of $\rho(u)$ as required.

To prove the correctness of the algorithm it suffices to remark that it determines a proof $g$ of the rank of $u$ such that $g_{i}>0$.

\subsection{Greedy algorithm on Dyck words}

Let $A$ be the alphabet with two letters $\{a, b\}$. For a word $w$ on the alphabet $A$ and for a letter $x \in A,|w|_{x}$ denotes the number of occurrences of $x$ in $w$. The function $\delta$ on words is defined by: $\delta(w)=|w|_{a}-|w|_{b}$. A Dyck word $w$ is a word on the alphabet $\{a, b\}$ such that $\delta(w)=0$, and for any of its prefixes $w^{\prime}$ one has $\delta\left(w^{\prime}\right) \geq 0$. The size of a Dyck word $w$ is $|w|_{a}=|w| / 2$. The height $h\left(w^{\prime}\right)$ of a prefix $w^{\prime}$ ending by an $a$ of a Dyck word $w$ is given by: $h\left(w^{\prime}\right)=\delta\left(w^{\prime}\right)-1$. The maximal height $H(w)$ of a Dyck word $w$ is $h(w)=\max _{w^{\prime}} h\left(w^{\prime}\right)$ where $w^{\prime}$ runs through all prefixes of $w$ ending with $a$.

To any (sorted) configuration $u$ of $K_{n}$ such that

$$
n-1 \geq u_{1} \geq u_{2} \geq \cdots u_{n-1} \geq 0
$$

we associate a word $w=D(u)$ with $n-1$ occurrences of $a$ and $n$ occurrences of $b$ the following way: the $i$ th occurrence of $a$ in $w$ has exactly $u_{n-i}$ occurrences of $b$ before it; notice that $D(u)$ ends

(i) We recall that configurations may have a negative number of tokens. 
with an occurrence of $b$. Moreover $D(u)$ is a Dyck word followed by a $b$, if and only if $u$ is a parking configuration. This leads to a reformulation of the preceding greedy algorithm in terms of Dyck words. When $u$ is a sorted parking configuration it is convenient to write $D(u)=d(u) b$ such that $d(u)$ is a Dyck word.

$\begin{array}{lllllll}5 & 4 & 4 & 2 & 0 & 0 & 0\end{array}$

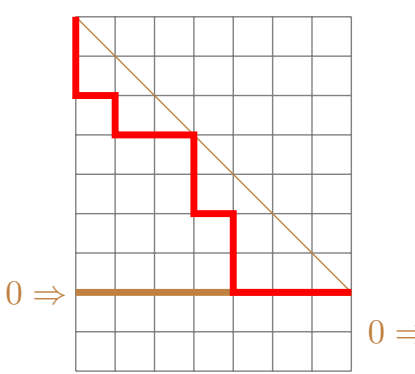

(a)
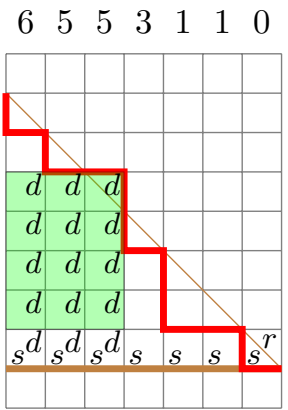

(b)

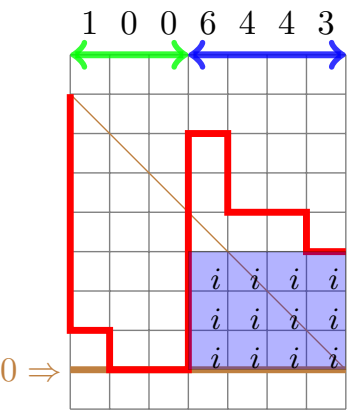

(c) $\begin{array}{lllllll}6 & 4 & 4 & 3 & 1 & 0 & 0\end{array}$

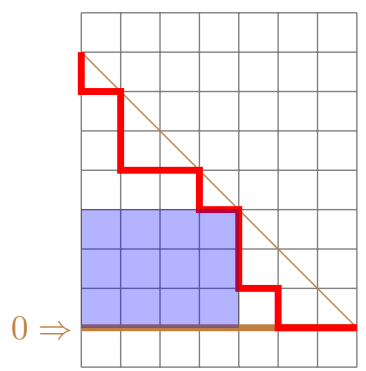

$(d)$

Fig. 3: An example of four steps of a loop iteration of algorithm computing rank

Any non-empty Dyck word $w$ admits the non-ambiguous classical first return decomposition $w=a$ fbg where $f$ and $g$ are Dyck words. As announced at the beginning of this Section, we consider the algorithm computing the rank in terms of sorted parking configurations toppling equivalent to it and its image via the preceding map $u \longrightarrow D(u)$. The algorithm may be described in terms of Dyck words due to:

Proposition 5 For any sorted parking configuration u, one step of the algorithm computing the rank consists in the subtraction of 1 on $u_{n-1}$ and then computing the sorted parking configuration $u^{\prime}$ toppling equivalent to it. In terms of words, this translates to the following: if $w=d(u)=$ afbg is the first return decomposition of $u$ then the new value of $w$ is $d\left(u^{\prime}\right)=g a b f$.

The algorithm is described in detail in the right part of Figure 2. We do not provide a detailed proof of Proposition 5 in this extended abstract, however we give details on an example of a loop iteration.

Assume that the algorithm reaches the sorted parking configuration $u=(5,4,4,2,0,0,0, s)$ for some $s \geq 0$, also described by $(d(u), s(u))=($ a aabbabbaababb, $s)$. We draw $d(u)$ in red from south-east to north-west in part $(a)$ of Figure 3 above. This red path and the brown horizontal axis pointed by $\Rightarrow 0$ define the diagram of the partition $\left(u_{1}, \ldots u_{n-1}\right)$ in which $u_{n}$ is omitted. We observe the following iteration step: we subtract 1 to $u_{n-1}$ and to recover positivity the vertex $x_{n}$ is toppled to reach $v=$ $(6,5,5,3,1,1,0, s-7)$. These two steps are represented in part $(b)$ of Figure 3. The cell labeled by $r$ describes the removed token and then the brown horizontal axis is lowered by one unit, adding one cell labeled by $s$ on each column of the partition which is the token coming from the toppling of the sink. This configuration $v$ is not parking since the three first vertices may topple together, preserving positivity. On (b), observe that it corresponds to the rightmost vertical cross of the red path with the brown diagonal, this should not be crossed if the configuration was a parking one. The toppling of the three first vertices leads to $w=(1,0,0,6,4,4,3, s-4)$ is illustrated in part $(c)$ of Figure 3 . The tokens transmitted from these 
three toppled vertices to the four untoppled vertices different from $x_{n}$ may be interpreted as those in cells labeled by $d$ in ( $b$ ) (before toppling) and by cells on (c) labeled by $i$ (after toppling). The configuration $w$ is sorted to get $w^{\prime}=(6,4,4,3,1,0,0, s-4)$ described in part $(d)$, and this sorting may be interpreted as taking a conjugate of the word $d(u)$. This sorting operation may also be also described by the exchange of $f$ and $g$ in the rewriting of $a f b g$ into gabf. In this example we have $d(u)=a f b g$ with $f=a a b b a b$ and $g=a a b a b b$ giving $g a b f=a a b a b b . a b . a a b b a b=d\left(w^{\prime}\right)$.

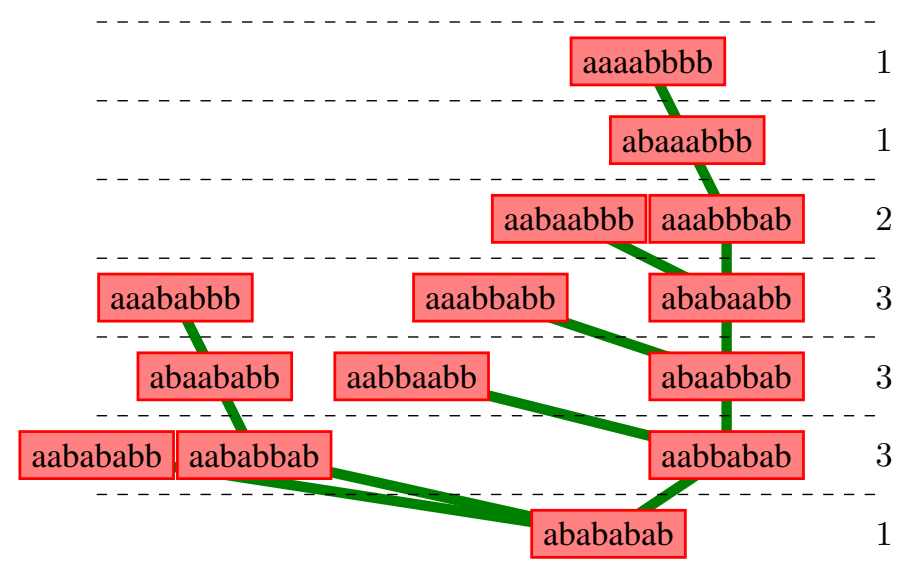

Fig. 4: The tree of Dyck words of size 4 describing the function $R$.

The rewriting $R(a f b g)=g a b f$ is a function on Dyck words of same size $n$ that may be described by a tree $T_{n}$ as in Figure 4 where edges $(w, R(w))$ are oriented downward. There is a loop not drawn at the root of the tree related to the single fixed point $R\left((a b)^{n}\right)=(a b)^{n}$. We define prerank $p(w)$ of any Dyck word as its distance to the root $(a b)^{n}$ or in other words $p(w)=\min \left\{k \mid k \geq 0\right.$ and $\left.R^{k}(w)=(a b)^{n}\right\}$. This is motivated by a count of the iterations required in the loop of the algorithm.

\subsection{Computing a parking configuration equivalent to $u$}

Lemma 2 Two configurations $u$ and $v$ are toppling equivalent in $K_{n}$ if and only if the following holds:

$$
\operatorname{deg}(u)=\operatorname{deg}(v) \text { and for any } 1 \leq i, j \leq n: u_{i}-u_{j}=v_{i}-v_{j}(\bmod n)
$$

Proof: It suffices to show that the configuration $u$ is toppling equivalent to 0 if and only if $\operatorname{deg}(u)=0$ and $u_{i}-u_{j}=0(\bmod n)$. But this follows from the fact that these relations are not modified by any toppling and are satisfied by the parking configuration equivalent to 0 which is equal to $(0,0, \ldots, 0)$.

Given a configuration $u$ one can find a configuration $v$ toppling equivalent to $u$ and such that $0 \leq v_{i}<n$ for any $1 \leq i \leq n-1$ by setting $v_{1}=0$, then $v_{i}=u_{i}-u_{1}(\bmod n)$ and $v_{n}=\operatorname{deg}(u)-\sum_{i=1}^{n-1} v_{i}$. From such a $v$ one builds the parking configuration using the following:

Proposition 6 Let u be a configuration satisfying equation (1) and let $w=D(u)$. The classical Cyclic Lemma states that there exists a unique conjugate $w^{\prime}$ of $w$ which is equal to a Dyck word followed by a 
letter $b$. Consider the configuration $v$ such that $D(v)=w^{\prime}$ and such that $v_{n}$ is such that $\operatorname{deg}(u)=\operatorname{deg}(v)$, then $v$ is the sorted version of the parking configuration equivalent to $u$.

\section{Symmetry of area and prerank distribution on Dyck words}

\subsection{A symmetry and a bijective proof of it}

The area of a Dyck word $w$ is defined by area $(w)=\sum_{w^{\prime}} h\left(w^{\prime}\right)$ where $w^{\prime}$ runs over all prefixes of $w$ ending with the letter $a$. We also consider for a Dyck word $w$ the largest prefix $u$ of it among those whose height is $H(w)$, and define the coheight $h^{c}\left(w^{\prime}\right)$ for any prefix $w^{\prime}$ of $w$ ending with an $a$, this coheight is $H(w)-h\left(w^{\prime}\right)$ if $w^{\prime}$ is not larger than $u$ and it is $H(w)-h\left(w^{\prime}\right)-1$ if $w^{\prime}$ is larger that $u$. Using Proposition 5 it is possible to prove that $\operatorname{prerank}(w)=\sum_{w^{\prime}} h^{c}\left(w^{\prime}\right)$ where $w^{\prime}$ runs over all prefixes of $w$ ending with the letter $a$.

We consider the generating function on Dyck words of size $n$ counted according to the statistics area and prerank:

$$
D_{n}^{\text {area,prerank }}(q, t)=\sum_{w} q^{\text {area }(w)} t^{\text {prerank }(w)} .
$$

Theorem 3 For any $n \geq 1$, we have the $\operatorname{symmetry~} D_{n}^{\text {area,prerank }}(q, t)=D_{n}^{\text {area,prerank }}(t, q)$.

The proof follows from an involution $\Phi$ on Dyck words that exchanges areas and preranks, and is defined as follows:

A non-empty Dyck word $w$ admits a non-ambiguous last maximum decomposition $w=u b v$ where $u$ is the largest prefix of $w$ among those whose height is $H(w)$. The mirror image $\tilde{w}$ of the word $w$ whose letters are $w_{1} w_{2} \ldots w_{k-1} w_{k}$ is the word $\tilde{w}=w_{k} w_{k-1} \ldots w_{2} w_{1}$; notice that we do not exchange letters $a$ and $b$. The involution $\Phi$ is defined from the last maximum decomposition $w=u b v$ by: $\Phi(u b v)=\tilde{u} b \tilde{v}$.

This symmetry can be refined at the level of occurrences of the letter $a$ in a Dyck word.

Lemma 3 For any Dyck word $w$ of size $n$ there is a bijection from the occurrences of the letter a in $w$ into those of the letter a in $\Phi(w)$ that exchanges heights and coheights. This bijection associates to an occurrence of a in $w$ its image by the involution $\Phi$.

The involution $\Phi$ has another property with respect to the dinv parameter introduced by Haiman (see Haglund (2008) for the definition of dinv).

Proposition 7 For any Dyck word $w$, $\operatorname{dinv}(\Phi(w))=\operatorname{dinv}(w)$.

An immediate corollary is that the bistatistic (prerank, dinv) is the image by $\phi$ of the bistatistic (area, dinv) which defines the $q, t$-Catalan numbers studied by A. Garsia, M. Haiman, J. Haglund.

Our definition of $\Phi$ may be seen, using mirror image, in the classical cyclic lemma attributed to Dvoretsky and Motzkin (1947). A word $w$ on the alphabet $\{a, b\}$ is called a quasi-balanced word of size $n$ if $|w|_{a}=n$ and $|w|_{b}=n+1$. The cyclic lemma states that for any quasi-balanced word $w$, among the $2 n+1$ conjugates of the bi-infinite periodic word $w^{\mathbb{Z}}$ exactly one may be written $\left(w^{\prime} b\right)^{\mathbb{Z}}$ where $w^{\prime}$ is a Dyck word of size $n$. The image of this via the mirror mapping is related to our definition of $\Phi$ : among the $2 n+1$ conjugates of $(\tilde{w})^{\mathbb{Z}}$ exactly one may be written $\left(w^{\prime \prime} b\right)^{\mathbb{Z}}$ where $w^{\prime \prime}$ is a Dyck word and $w^{\prime \prime}=\Phi\left(w^{\prime}\right)$. 
It is also possible to prove that the involution $\Phi$ on Dyck paths satisfies a commutativity relation with the function $\zeta$ introduced in Haglund (2008) (page 50). More precisely : Flip. $\zeta=\zeta \Phi$, where Flip is the map that reflects a Dyck word and exchanges occurrences of $a$ 's and $b$ 's (ii)

\subsection{Another description of the rank algorithm}

The conjugate $\Phi R \Phi$ of function $R$ with this bijection $\Phi$ is described by the following lemma which leads to another description of the rank algorithm.

Lemma 4 For any non-empty Dyck word $w$, let $\Phi(w)=u b v=\left(u^{\prime} a\right) b v$ be the last maximum decomposition of $\Phi(w)$ then $\Phi(R(w))=u^{\prime}$ bav.

The building of the tree in Fig. 4 becomes obvious from this viewpoint, when the nodes of $T_{n}$ are labeled by $\Phi(w)$ instead of $w$ since the rewriting described by the edge $(\Phi(d), \Phi(R(d)))$ corresponds to a flip of the last highest peak $a b$ into a valley $b a$.

\subsection{Computing the area, prerank distribution}

We currently have two ways to describe the distribution of the bistatistic (area,prerank) on Dyck words of given size $n$. First, we have a non-ambiguous shuffle of any possible distribution of pairs heights and coheights on occurences of letter $a$ leading to all Dyck words with this distribution:

Proposition 8 For any $n \geq 0$ and $k$ such that $1 \leq k \leq n$, let $c=\left(c_{0}, c_{1}, \ldots c_{2 k-2}\right)$ be a composition of $n-k$ into $2 k-1$ parts. The number $N_{n, k, c}$ of Dyck words such that $1+c_{2 i}$ is the number of letters a of height $i$ and coheight $k-i$ and $c_{2 i+1}$ is the number of letters $a$ of height $i$ and coheight $k-1-i$ is

$$
N_{n, k, c}=\prod_{i=0}\left(\begin{array}{c}
c_{2 i}+c_{2 i+2} \\
c_{2 i}
\end{array}\right)\left(\begin{array}{c}
c_{2 i+1}+c_{2 i+3} \\
c_{2 i+1}
\end{array}\right)
$$

consequently,

$$
D_{n}^{\text {area,prerank }}(q, t)=\sum_{k=1}^{n} \sum_{c \text { composition of } n-k} N_{n, k, c} \prod_{i=0}^{k}\left(q^{i} t^{k-i}\right)^{1+c_{2 i}}\left(q^{i} t^{k-1-i}\right)^{c_{2 i+1}} .
$$

Using an interpretation of the decomposition at last maximum of the Dyck word in terms of heaps of dimers in the framework of Viennot's theory of heaps (see Krattenthaler (2006) ) we also have:

Lemma 5 Let $\left(T_{n}(y, z)\right)_{n \geq 0}$ the polynomials recursively defined by $T_{0}(y, z)=1=T_{1}(y, z)$ and for $n \geq 2$,

$$
T_{n}(y, z)=T_{n-1}(y, z)+y^{n-2} z T_{n-2}(y, z)
$$

then

$$
\sum_{n \geq 1} D_{n}^{\text {area,prerank }}(q, t) z^{n}=\sum_{k \geq 2} \frac{(q t)^{\left(\begin{array}{c}
k-1 \\
2
\end{array}\right)} z^{k-1}}{T_{k}\left(q / t, t^{k-2} z\right) T_{k-1}\left(q / t,-t^{k-3} z\right)} .
$$

(ii) We thank one of the anonymous referees of FPSAC 2013 to have suggested the existence of this link 


\section{On degree and rank distribution}

\subsection{On any graph $G$}

Given a sink, labeled by $n$ in our notation, the toppling classes of configurations may be indexed by $G$-parking configurations $(\pi, s)$ where $\pi$ belongs to $\Pi_{G}$ the finite set of restrictions of $G$-parking configurations outside the sink and $s \in \mathbb{Z}$ is a number of tokens on the sink. These indices are used to define the Laurent series related to the distribution of degree and rank by

$$
P_{G}^{\text {degree }, \text { rank }}(x, r)=\sum_{\pi \in \Pi_{G}, s \in \mathbb{Z}} x^{\text {degree }((\pi, s))} r^{\operatorname{rank}((\pi, s))} .
$$

Since a negative degree implies a rank equal to -1 , using Proposition 4 for higher degrees we can consider that the relevant part of this series is a ("Laurent") polynomial $P_{G,[0,2 m-2 n]}^{\text {degree,rank }}(x, r)$ defined on configurations with intermediate degree, that is belonging to the interval $[0,2 m-2 n]$. Hence we write:

$$
P_{G}^{\text {degree, rank }}(x, r)=\frac{(r x)^{-1}\left|\Pi_{G}\right|}{1-x^{-1}}+P_{G,[0,2 m-2 n]}^{\text {degree,rank }}(x, r)+\frac{x\left(x^{2} r\right)^{m-n}\left|\Pi_{G}\right|}{1-x r} .
$$

Theorem 2 uses configuration $\kappa$ of degree $2 m-2 n$ to give a relation between the rank and degree of two configurations $u$ and $\kappa-u$, it implies the following formula expressing symmetry of degree and rank distribution:

$$
P_{G}^{\text {degree,rank }}(x, r)=\left(r x^{2}\right)^{m-n} P_{G}^{\text {degree,rank }}\left(\frac{1}{x r}, r\right) .
$$

The non-effective configurations are exactly those of rank -1 and the degree distribution on these configurations may be related to an evaluation of the Tutte polynomial $T_{G}(x, y)$ of the graph $G$ (see Lopez (1997)) where $x$ (respectively $y$ ) counts internal (respectively external) activity:

$$
\left[r^{-1}\right] P_{G}^{\text {degree,rank }}(x, r)=\frac{1}{1-x^{-1}} T_{G}(1, x) .
$$

\subsection{On complete graphs}

In the particular case the complete graph $K_{n}, m=\left(\begin{array}{l}n \\ 2\end{array}\right)$, we define the distribution of degree and rank at the level of orbits under the action of $S_{n-1}$ leading to the "Laurent" polynomial:

$$
D_{n}^{\text {degree }, \text { rank }}(x, r)=\sum_{u} x^{\text {degree }(u)} r^{\text {rank }(u)},
$$

where $u$ runs over sorted parking configurations such that degree $(u) \in[0, n(n-3)]$.

Baker and Norine's theorem is compatible with the action of $S_{n-1}$ so we also have the symmetry

$$
D_{n}^{\text {degree }, \text { rank }}(x, r)=\left(r x^{2}\right)^{n(n-3) / 2} D_{n}^{\text {degree, rank }}\left(\frac{1}{x r}, r\right) .
$$

We conclude this extended abstract by the partial announcement of an enumerative result we obtained recently via combinatorial considerations on the analysis of our algorithm computing the rank. This can be stated as follows:

$$
D_{n}^{\text {degree, }, \text { rank }}(x, r)=x^{\left(\begin{array}{c}
n-1 \\
2
\end{array}\right)-1} r^{-1}\left(\left[z^{n}\right] F\left(q_{1}, q_{2} ; z\right)\right)
$$


where $\left[z^{n}\right] f(z)$ denotes the coefficient of $z^{n}$ in power sum $f(z)$, and $F\left(q_{1}, q_{2} ; z\right)$ is an explicit rational function in $q_{1}=x^{-1}, q_{2}=x r, z, C\left(q_{1} ; z\right), C\left(q_{1} ; q_{1} z\right), C\left(q_{2} ; z\right)$ and $C\left(q_{2} ; q_{2} z\right)$ where

$$
C(q ; z)=\sum_{w d y c k} q^{\operatorname{area}(w)} z^{\text {size }(z)}
$$

is the well known Carlitz $q$-analogue of Catalan numbers.

\section{References}

O. Amini and M. Manjunath. Riemann-Roch for sub-lattices of the root lattice $A_{n}$. Electronic J. of Combinatorics, 17:R124, 2010.

P. Bak, C. Tang, and K. Wiesenfeld. Self-organised criticality. Physical Review A., 38:364-374, 1988.

M. Baker and S. Norine. Riemann-Roch and Abel-Jacobi theory on a finite graph. Advances in Mathematics, 215:766-788, 2007.

N. Biggs. Chip-firing and the critical group of a graph. J. of Algebraic Combinatorics, 9:25—45, 1999.

A. Björner, L. Lovász, and P.Shor. Chip-firing games on graphs. European J. Combin, 12:283-291, 1991.

R. Cori and D. Rossin. On the sandpile group of dual graphs. Europ. J. Comb, 21:447—459, 2000.

D. Dhar. Self-organized critical state of the sandpile automaton models. Phys. Rev. Lett., 64:1613-1616, 1990.

D. Dhar and S. Majumdar. Equivalence between the Abelian sandpile model and the $q \rightarrow 0$ limit of the Potts model. Physica A, 185:129-135, 1992.

A. Dvoretsky and T. Motzkin. A problem of arrangements. Duke Math. J., 14:305—313, 1947.

H. M. Farkas and I. Kra. Riemann Surfaces. Graduate Texts in Mathematics, Springer, 1992.

A. Garsia and M. Haiman. A remarkable $q, t$-catalan sequence and $q$-lagrange inversion. J. Algebraic Combin., 5:191-244, 1996.

J. Haglund. The q,t-Catalan numbers and the space of diagonal harmonics. AMS University Lectures Series, 2008.

C. Krattenthaler. The theory of heaps and the Cartier-Foata monoid. available at: http://www.mat.univie.ac.at/ slc/books/heaps.ps, 2006.

C. M. Lopez. Chip firing and Tutte polynomials. Ann. Combin., 3:253-259, 1997.

M. Manjunath. The rank of a divisor on a finite graph: geometry and computation. preprint, arXiv:1111.7251, 2011. 